\title{
PSYCHOPHYSIOLOGICAL RESPONSES OF WOMEN WITH POLYCYSTIC OVARY SYNDROME DURING SESSIONS OF HIGH-INTENSITY INTERVAL TRAINING
}

original paper

() Wroclaw University of Health and Sport Sciences

DOI: https://doi.org/10.5114/hm.2022.108315

\section{ISIS KELLY DOS SANTOS ${ }^{1 \oplus}$, LUIZ FERNANDO FARIAS-JUNIOR ${ }^{2 \oplus}$, GABRIEL SOARES PICHINI ${ }^{1}$, CARLINDO DANIEL DE MEDEIROS LOPES FERREIRA ${ }^{1}$, VICTOR SABINO QUEIROS ${ }^{1 \oplus}$, GUSTAVO MAFALDO SOARES ${ }^{3}$, BRENO GUILHERME DE ARAÚJO TINOCO CABRAL ${ }^{1 \oplus}$, TECIA MARIA DE OLIVEIRA MARANHÃ ${ }^{3}$, PAULO MOREIRA SILVA DANTAS ${ }^{1}{ }^{\circledR}$}

${ }^{1}$ Graduate Program in Health Sciences, Federal University of Rio Grande do Norte, Natal, Brazil

${ }^{2}$ Graduate Program in Psychobiology, Federal University of Rio Grande do Norte, Natal, Brazil

${ }^{3}$ Department of Toco-Gynaecology, Federal University of Rio Grande do Norte, Natal, Brazil

\begin{abstract}
Purpose. The study described psychophysiological responses (perceived exertion, affective response) of women with polycystic ovary syndrome (PCOS) throughout 10 sessions of high-intensity interval training (HIIT).

Methods. Overall, 12 physically inactive women with PCOS (age: $26.2 \pm 4.1$ years; body mass index: $28.5 \pm 6.3 \mathrm{~kg} / \mathrm{m}^{2}$ ) performed 10 running HIIT sessions of $50 \mathrm{~min}$ [10 $\times 1$-min intervals, 90\% of maximal heat rate (HRmax); 3-min active recovery periods, 70\% HRmax], including 5-min warm-up and cool-down, with 48-hour intervals. HRmax, rating of perceived exertion (RPE, Borg CR10 scale), and affective responses (Feeling Scale) were assessed in the last $10 \mathrm{~s}$ of each interval and recovery period. Each session responses were grouped into session parts (beginning, 1-3; middle, 4-7; end, 8-10) for intervals and recovery periods separately. The generalized estimating equation model served to compare \%HRmax, RPE, and affective valence between session parts.

Results. The intensities of intervals $(p<0.001)$ and recovery $(p<0.001)$ increased from session beginning to end, as did $\mathrm{RPE}$ of intervals $(p<0.001)$ and recovery $(p<0.001)$. Interval affective responses were lower at end compared with beginning $(p<0.040)$. HIIT intensity (\%HRmax) presented a small coefficient of variation (CV, ca. 3\%); RPE exhibited medium CV (ca. 20\%) between 10 HIIT sessions.

Conclusions. Psychophysiological responses of physically inactive PCOS women to running HIIT demonstrate positive affective valence at the beginning of HIIT sessions. As the session progresses, affective valence decreases to neutral and RPE increases.
\end{abstract}

Key words: PCOS, interval training, affect, pleasure, perceived exertion

\section{Introduction}

High-intensity interval training (HIIT) is a physical exercise approach characterized by repeated high-intensity bouts performed above $85 \%$ of maximal heat rate (HRmax) interspersed with passive or active recovery periods [1, 2]. Research has demonstrated the efficacy of HIIT to promote improvement in physical fitness (e.g., cardiorespiratory fitness) and health pa- rameters (e.g., blood pressure and glycaemia) in adults predisposed to established hypertension or overweight/ obesity [3-5]. Moreover, including HIIT in training programs for women with polycystic ovary syndrome (PCOS) showed greater health-enhancing benefits $[6,7]$. PCOS is the most common endocrinopathy that appears in reproductive age [8] and presents elevated risk of developing insulin resistance, dyslipidaemia, and obesity [9]. Typically, women with PCOS present

Correspondence address: Paulo Moreira Silva Dantas, Department of Physical Education, Federal University of Rio Grande do Norte, BR 101, Lagoa Nova, Natal RN 59.078-970, Brazil, e-mail: pgdantas@ufrnet.br, https://orcid.org/0000-0002-9217-7107

Received: December 18, 2020

Accepted for publication: March 17, 2021

Citation: Dos Santos IK, Farias-Junior LF, Pichini GS, De Medeiros Lopes Ferreira CD, Queiros VS, Soares GM, De Araújo Tinoco Cabral BG, De Oliveira Maranhão TM, Dantas PMS. Psychophysiological responses of women with polycystic ovary syndrome during sessions of high-intensity interval training. Hum Mov. 2022;23(4):112-122; doi: https://doi.org/10.5114/ hm.2022.108315. 
metabolic syndrome [10], which increases the risk for diabetes mellitus and cardiovascular disease [11].

Lifestyle modifications such as regular exercise practice and healthy eating are non-drug treatment recommendations for women with PCOS [12-14]. Moderate-intensity aerobic exercise improves the healthrelated quality of life, cardiorespiratory fitness, and cardiometabolic profile of overweight/obese women with PCOS [15]. Additionally, high-intensity aerobic exercise improves insulin resistance in women with PCOS [16]. Recently, Samadi et al. [16] demonstrated that 12 weeks $(3 \times /$ week for $20 \mathrm{~min}$ ) of aquatic HIIT (i.e., $4 \times 4$-min bouts, each consisting of 8 rounds of 20 -s all-out exertion interspaced with $10 \mathrm{~s}$ of rest) together with the use of metformin $(1500 \mathrm{mg}$ ) reduced body mass index (BMI), fat mass, and insulin resistance, and improved the levels of reproductive hormones in women with PCOS. Additionally, Aktaş et al. [17] indicated that $3 \times /$ week for 12 weeks of HIIT (e.g., 7 intervals of 2-min running interspaced with 2-min walking) compared with moderate continuous training (e.g., 30-min running at a constant speed) reduced the levels of insulin, total triglycerides, total cholesterol, and LDL-cholesterol, and increased the levels of adiponectin and HDL-cholesterol in women with PCOS.

These findings demonstrate the efficacy of HIIT to promote improvement in health parameters in women with PCOS, but psychophysiological responses (e.g., affective valence and perceived exertion) of these women during HIIT is not known [7, 18]. Psychophysiological responses are important factors to take into account when prescribing physical exercise in order to encourage adherence to regular practice [18], particularly in women with PCOS who are overweight or obese. From a psychological perspective, individuals with excess weight are more likely to present increased perceived exertion and reduced affective response than their peers of normal weight [19], factors that reduce the exercise practice time at 6 and 12 months [20-22]. Previously, Decker and Ekkekakis [23] showed that the affective valence became negative at the end of the HIIT session in women with obesity who performed a single HIIT session with high interval/recovery ratio (i.e., 3:2 interval/recovery time) [23]. This relationship is suggested to be due to the high-intensity nature of HIIT [23]. According to the dual model theory, aerobic exercise performed above the respiratory compensatory point (i.e., high-intensity) elicits negative affective valence [24]. Previous studies have demonstrated an increase in the perception of effort and a decrease in the affective response during HIIT sessions [25-27]. Therefore, it is expected that physically inactive women with
PCOS present decreased affective valence throughout the HIIT session. However, the intermittent nature of HIIT allows individuals to recover from intervals of high-intensity during recovery periods and it is possible to draw up different HIIT protocols [28] which are more tolerable. HIIT protocols with lower interval/ recovery ratio are suggested as being more tolerable [29], an example being an HIIT protocol with 1:3 inter$\mathrm{val} /$ recovery ratio. Moreover, psychophysiological responses (i.e., affective valence and perceived exertion) of women with PCOS during subsequent HIIT sessions are not known. Therefore, the aim of this study was to describe the psychophysiological responses (i.e., perceived exertion and affective responses) of women with PCOS throughout 10 sessions of HIIT involving running. We hypothesized that during the HIIT sessions, the perceived exertion would increase and affective valence would decrease, in line with previous studies [25-27], while the psychophysiological responses throughout 10 HIIT running sessions would remain unchanged.

\section{Material and methods}

\section{Study design}

This cross-sectional descriptive study involved 12 women with PCOS aged $18-40$ years $(26.2 \pm 4.1)$. All participants were informed about all study procedures. The study followed the recommendations of the STROBE checklist in reporting cross-sectional studies [30].

\section{Setting}

The study was divided into sections and the participants underwent: (i) an initial screening of clinical health and anthropometric and body composition evaluation; (ii) a familiarization procedure (i.e., 3 exercise sessions in the first week); and (iii) 10 running HIIT sessions with at least 48-hour intervals. We recruited women with PCOS from the outpatient clinic of the endocrinology-gynaecological Maternidade Escola Januario Cicco hospital hospital between January 2018 and June 2019 during medical consultations or by using social media.

\section{Participants}

A total of 125 women with PCOS were recruited at first; 82 women were assessed for eligibility, and only 15 patients diagnosed with PCOS participated in all 


\section{HUMAN MOVEMENT}

I.K. Santos et al., Training responses of women with polycystic ovary syndrome

phases of the study. However, 3 participants were excluded from the analysis because they reported the same affective responses during the exercise sessions in all 10 running HIIT sessions (Figure 1). The diagnosis of PCOS was established by a gynaecologist by using the Rotterdam criteria [31]. After the consultation, all participants responded to the Physical Activity Readiness Questionnaire (PAR-Q) [32] and the International Physical Activity Questionnaire (IPAQ), short version [33]. PAR-Q was used to verify the women's state of readiness for physical exercise, IPAQ assessed their physical activity level (e.g., walking, moderate-intensity activities, and vigorous-intensity activities).

The inclusion criteria were: (i) age of 18-40 years; (ii) a diagnosis of PCOS; and (iii) no practice of exercise or having been inactive within the previous 6 months. The exclusion criteria involved: (i) contraindication for physical exercise; (ii) diagnosis of cardiovascular or orthopaedic diseases; (iii) $\mathrm{BMI} \leq 18.5 \mathrm{~kg} / \mathrm{m}^{2}$ or $\geq 40.0 \mathrm{~kg} / \mathrm{m}^{2}$; and (iv) 2 consecutive absences in the protocol familiarization phase.

\section{Variables}

\section{Initial screening}

Initially, the participants underwent a screening of health. Afterwards, body mass (kg) and height (m) were measured, and BMI was calculated. The BMI was classified in accordance with the World Health Organization criteria (i.e., normal weight: $18.5-24.9 \mathrm{~kg} / \mathrm{m}^{2}$; overweight: $25-29.9 \mathrm{~kg} / \mathrm{m}^{2}$, and obesity: $\geq 30 \mathrm{~kg} / \mathrm{m}^{2}$ ) [34]. The waist and hip circumference were measured [35]. The waist-to-hip ratio was calculated and classified in accordance with the World Health Organization criteria (i.e., $\geq 0.85$ for females is defined as risk of cardiovascular disease) [35]. The measure-

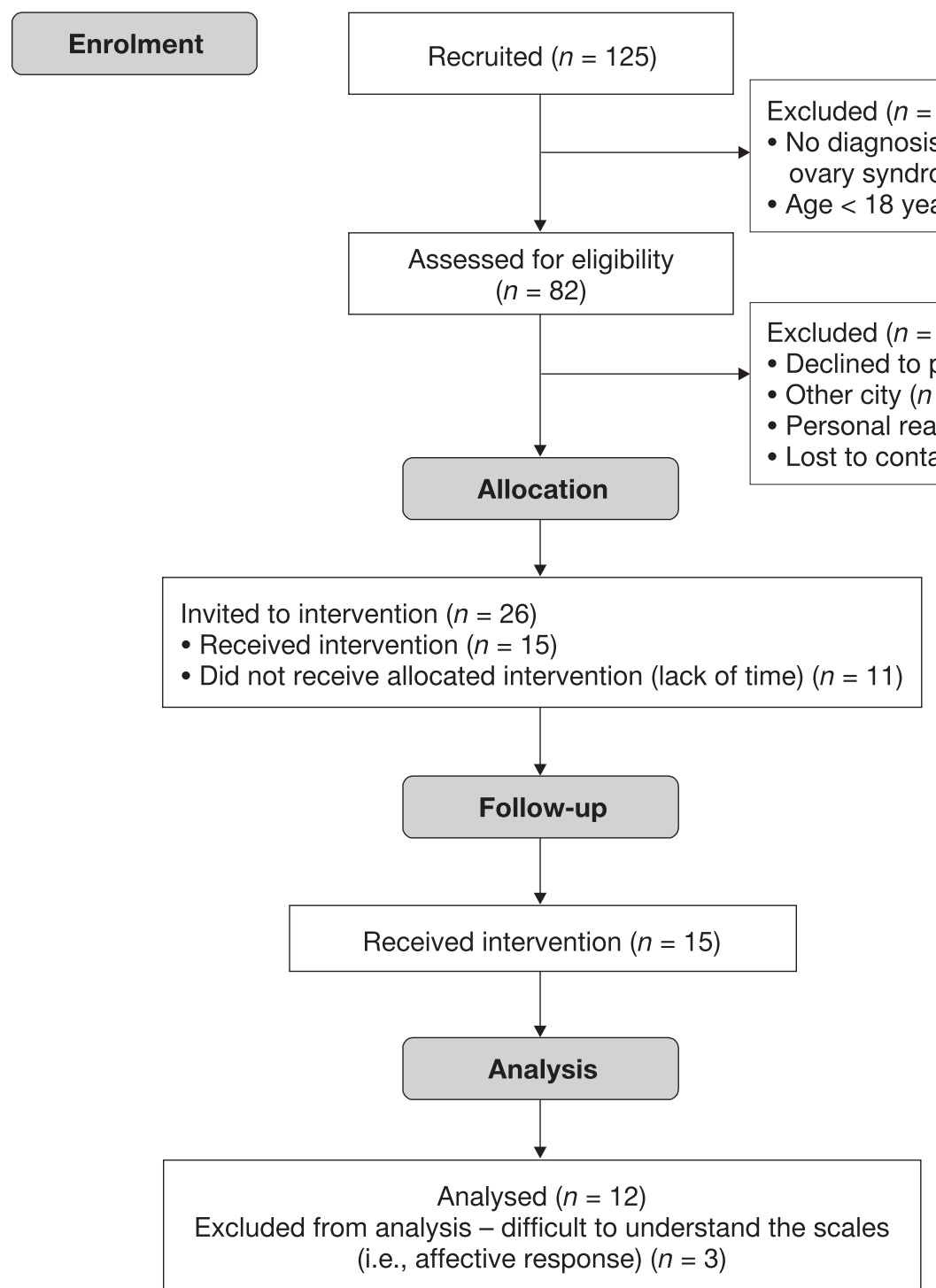

Figure 1. Flow chart of the study 
ments were performed by an experienced evaluator who followed all recommendations by the International Society for the Advancement of Kinanthropometry [36]. Subsequently, the participants remained lying supine in a silent room with a controlled temperature $\left(23-26^{\circ} \mathrm{C}\right.$ ) in order to measure the resting HR (Polar FT1, Polar Electro, Kempele, Finland). Then, blood pressure was evaluated 3 times with an interval of 1 min by using the oscillometric method (Omron ${ }^{\circledR}$ HEM7200, Omron, USA). The mean of the 2 last measurements was considered resting blood pressure [37]. Finally, body composition was measured with dualenergy X-ray absorptiometry (GE Healthcare ${ }^{\circledast}$ Lunar Prodigy Advance, GE Healthcare, Madison, USA).

\section{Affective valence}

The Feeling Scale is a bipolar scale of 11 points consisting of negative and positive valences, such as: $-5=$ very bad; $-3=$ bad; $-1=$ quite bad; $0=$ neutral; $+1=$ reasonably good; $+3=$ good; $+5=$ very good. Immediately prior to the 3 familiarization HIIT sessions, the meaning of basic affective valence was explained to the patients in accordance with the instructions provided by Hardy and Rejeski [38]: 'While participating in exercise it is quite common to experience changes in mood. Some individuals find exercise pleasurable, whereas others find it to be unpleasurable' (p. 308). Additionally, feeling may fluctuate across time. That is, one might feel good and bad a number of times during exercise. Scientists have developed a scale to measure such responses (at this point, the participants were presented with a copy of the Feeling Scale). Affective valence was defined as the subjective feeling of the 'pleasure' or 'displeasure' during exercise that occurs regardless of emotions [38, 39]. A validated scale is applied to measure basic affect during physical exercise [40]. In adult males and females aged 19-63 years, the Feeling Scale has a test-retest intraclass correlation coefficient equal to 0.644 (95\% CI: 0.970-0.985; typical error: 1.1, 95\% CI: 0.94-1.3; coefficient of variation: $24.4 \%$ ) [40]. These subjective feelings were explained in the theoretical anchorage procedure, and they were established during the 3 familiarization HIIT sessions. During the exercise session, the participants were asked 'How do you feel at this moment of the exercise session?' and were presented with a scale.

\section{Rating of perceived exertion}

The Borg rating of perceived exertion (RPE) category-ratio (CR10) scale [41] is validated to measure the perception of effort during physical exercise [42]. Immediately prior to the 3 familiarization HIIT sessions, the meaning of perceived exertion was explained to the participants. Perceived exertion was defined as the subjective intensity of effort, strain, and/or fatigue perceived during exercise [43]. Low and high perceptual anchors for the Borg RPE CR10 scale were explained in the theoretical anchorage procedure, and they were established during the 3 familiarization HIIT sessions. A rating of 0 (low anchor, 'nothing at all') was assigned to the lowest exercise intensity, while a rating of 10 (high anchor, 'extremely strong') was assigned to the highest exercise intensity [41]. During the exercise sessions, the women were asked 'What is your perceived exertion at this moment of the exercise session?' and were presented with a scale.

\section{Familiarization procedure}

The participants performed 3 sessions with a 48hour interval supervised by a physical exercise professional in order to familiarize themselves with the Feeling Scale [38], the Borg RPE CR10 scale [41], and the running HIIT protocol. Initially, in the familiarization sessions, the patients were individually instructed about both scales. A theoretical anchorage procedure was used, encouraging the subjects to imagine several previous physical exercise experiences when they perceived exertion as light, moderate, and extremely strong, and when they felt physical exercise as pleasure and displeasure. Furthermore, the participants were instructed regarding the time and intensity of intervals and recovery of the running HIIT protocol. Each familiarization session lasted for $50 \mathrm{~min}$ and consisted of theoretical anchorage procedures, warm-up, a reduced HIIT session (i.e., $35 \mathrm{~min}$ ), and cool-down. During HIIT sessions, HR, RPE, and affective valence were recorded in the last $10 \mathrm{~s}$ of each interval and recovery. The scales used to measure affective valence and RPE were shown to the women in a randomized order. The HR, RPE, and affective valence responses in each exercise session were grouped into session parts (beginning, 1-3; middle, 4-7; and end, 8-10) for intervals and recovery periods separately.

\section{HIIT protocol}

All running HIIT sessions were performed on a 400-m running track. The exercise sessions lasted for $50 \mathrm{~min}$, including $5 \mathrm{~min}$ of warm-up at $60-65 \%$ of age-predicted HRmax (220 - age), 40 min of a HIIT running protocol, and 5 min of cool-down. The run- 
ning HIIT protocol consisted of 10 intervals of $1 \mathrm{~min}$ at 95\% HRmax interspaced with 10 active recovery periods of 3 min at 70\% HRmax. This HIIT protocol was adopted because its 1:3 interval/recovery ratio is more tolerable and its total volume of $40 \mathrm{~min}$ together with the active recovery allows for high caloric expenditure per session [1]. All participants were instructed about their intensity of interval and recovery. They were asked to run during the intervals and to walk during recovery in order to reach the intensity target. HR was continuously monitored with a HR monitor system (Polar FT1, Polar Electro, Kempele, Finland). The attendance in the sessions was recorded.

\section{Statistical analyses}

The data normality was verified with the ShapiroWilk test and $z$-scores of asymmetry and kurtosis (-1.96 to 1.96). The parametric data are expressed as mean and standard deviation, while the non-parametric data are expressed as median and $25^{\text {th }}$ and $75^{\text {th }}$ percentiles. A generalized estimating equation (GEE) model was used to compare the participants' HR, RPE, and affective valence responses between session parts (i.e., beginning, middle, and end). For the HR and RPE responses, the gamma distribution GEE model was applied with an identity link based on the goodness of fit, while for the affective valence, a linear distribution was used because of negative values. The goodness of fit of the models was verified by the normal Q-Q plot and likelihood under the independence model criterion. Coefficients of variation for HR and RPE between 10 sessions for each session part (i.e., beginning, middle, and end) were calculated. The coefficient of variation for affective valence was not calculated because of its negative values. An assumption of this analysis is that all values were positive. Cohen's $d$ was used to verify the effect size $(E S)$ for comparisons within session parts [44]. The following criteria for $E S$ interpretation were adopted: $<0.50$, small; 0.50-0.79, medium; and $\geq 0.80$, large [45]. For all analyses, a statistical significance of 0.05 was assumed. Data were analysed with the IBM SPSS Statistics for Win/v.25.0 (IBM Corp., Armonk, NY, USA).

\section{Ethical approval}

The research related to human use has complied with all the relevant national regulations and institutional policies, has followed the tenets of the Declaration of Helsinki, and has been approved by the authors' Institutional Ethics Committee of Federal University of Rio Grande do Norte (protocol number 1.863.259/2016).

\section{Informed consent}

Informed consent has been obtained from all individuals included in this study.

\section{Results}

The attendance rate of the HIIT sessions was 100\% and no adverse events occurred over the 10 HIIT sessions or were reported by participants during the intervention periods. The body composition and clinical characteristics of the subjects are presented in Table 1. A total of $58 \%$ of the women $(n=7)$ were overweight or obese. All obese participants $(n=5)$ had elevated waist circumference of $101.0 \pm 3.7 \mathrm{~cm}$. The patients exhibited a low physical activity level (they were physically inactive) and increased sitting time during the week and weekend. All participants had a normal systolic and diastolic blood pressure.

The comparison of participants' HR, RPE, and affective valence responses between session parts (i.e., beginning, middle, and end) throughout the 10 HIIT sessions are presented in Table 2.

Table 1. Participants' characteristics $(n=12)$

\begin{tabular}{lc}
\hline Variables & $\begin{array}{c}\text { Mean } \pm S D \\
\text { or median } \\
\text { (interquartile range) }\end{array}$ \\
\hline Age (years) & $26.2 \pm 4.1$ \\
Body mass (kg) & $75.8 \pm 17.1$ \\
Height (m) & $1.63 \pm 0.07$ \\
Body mass index (kg/m $\left.{ }^{2}\right)$ & $28.5 \pm 6.3$ \\
Waist circumference (cm) & $86.2 \pm 13.7$ \\
Hip circumference (cm) & $107.5 \pm 11.9$ \\
Waist-to-hip ratio (\%) & $0.80 \pm 0.07$ \\
Free lean mass (kg) & $42.4 \pm 7.6$ \\
Total fat (\%) & $40.3 \pm 6.1$ \\
Trunk fat (\%) & $41.9 \pm 6.7$ \\
Android fat (\%) & $44.0 \pm 8.6$ \\
Gynoid fat (\%) & $44.6 \pm 6.8$ \\
Systolic blood pressure (mm Hg) & $113.3 \pm 9.8$ \\
Diastolic blood pressure (mm Hg) & $78.3 \pm 9.4$ \\
Resting heart rate (bpm) & $64.6 \pm 6.1$ \\
Estimated maximum heart rate (bpm) & $193.8 \pm 4.1$ \\
\hline Physical activity level (International Physical \\
Activity Questionnaire) \\
Walking (min/week) \\
Moderate intensity (min/week) \\
Vigorous intensity (min/week) \\
\hline Time sitting & $0.0(0.0 ; 10.0)$ \\
$\quad$ Week (hours/day) & $0.0(0.0 ; 0.0)$ \\
\hline
\end{tabular}


I.K. Santos et al., Training responses of women with polycystic ovary syndrome

Table 2. Percentage of maximum HR, RPE, and affective responses by parts of sessions throughout the 10 running high-intensity interval training sessions $(n=12)$

\begin{tabular}{|c|c|c|c|c|c|c|c|}
\hline \multirow{2}{*}{ Variables } & \multicolumn{6}{|c|}{ Parts of session } & \multirow{2}{*}{$p$} \\
\hline & Beginning & CV (\%) & Middle & CV (\%) & End & CV (\%) & \\
\hline \multicolumn{8}{|l|}{ Intervals } \\
\hline Maximum HR (\%) & $92.9 \pm 5.5$ & 4 & $94.5 \pm 4.8^{*}$ & 3 & $96.6 \pm 4.4^{* \#}$ & 3 & $<0.001$ \\
\hline RPE (0-10) & $4.6 \pm 1.2$ & 23 & $6.2 \pm 1.2^{*}$ & 16 & $7.2 \pm 1.4^{* \#}$ & 16 & $<0.001$ \\
\hline Affective response $(-5 /+5)$ & $1.4 \pm 1.6$ & - & $0.6 \pm 2.2$ & - & $0.2 \pm 2.2^{*}$ & - & 0.040 \\
\hline \multicolumn{8}{|l|}{ Recovery } \\
\hline Maximum HR (\%) & $74.2 \pm 4.7$ & 4 & $76.7 \pm 4.4^{*}$ & 4 & $77.4 \pm 4.6^{* \#}$ & 4 & $<0.001$ \\
\hline RPE (0-10) & $3.0 \pm 1.1$ & 25 & $4.8 \pm 1.4^{*}$ & 21 & $5.5 \pm 1.6^{* \#}$ & 18 & $<0.001$ \\
\hline Affective response $(-5 /+5)$ & $2.1 \pm 1.5$ & - & $1.2 \pm 2.1$ & - & $0.6 \pm 2.2^{* \#}$ & - & $<0.001$ \\
\hline
\end{tabular}

Data expressed as mean \pm stand deviation.

$\mathrm{CV}$ - intra-session coefficient of variation, HR - heart rate, RPE - rating of perceived exertion

* statistically different from the beginning, \# statistically different from the middle
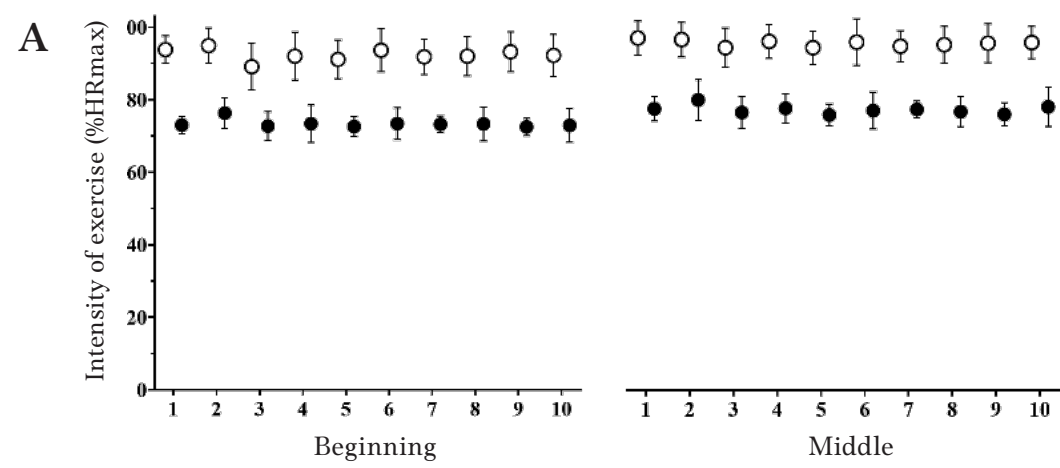

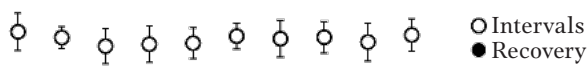

B
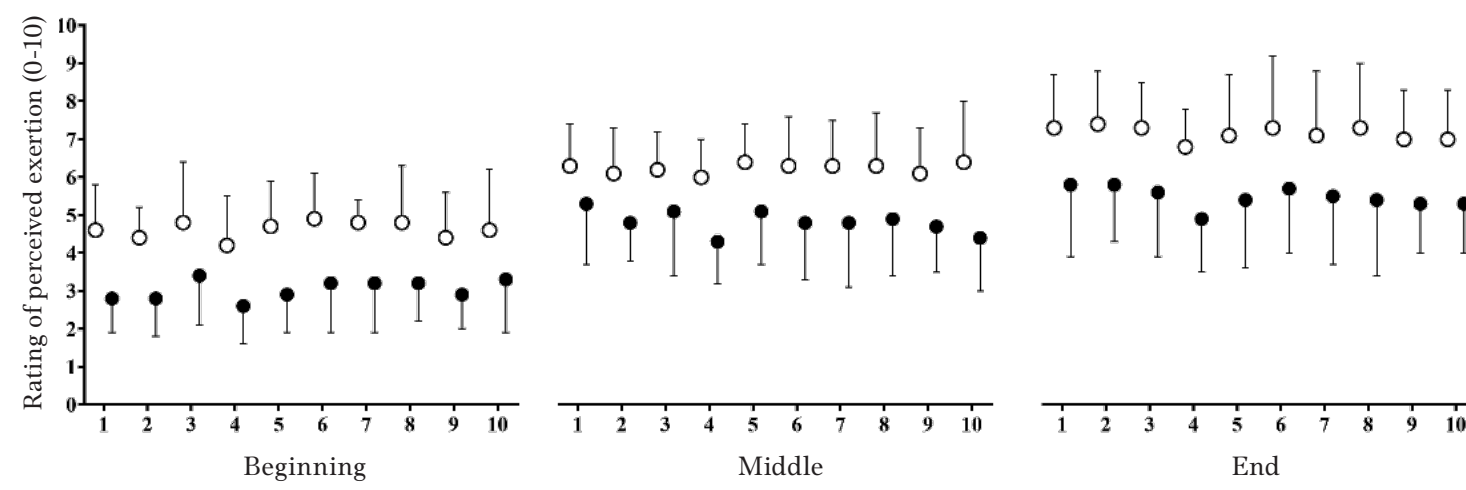

O Intervals
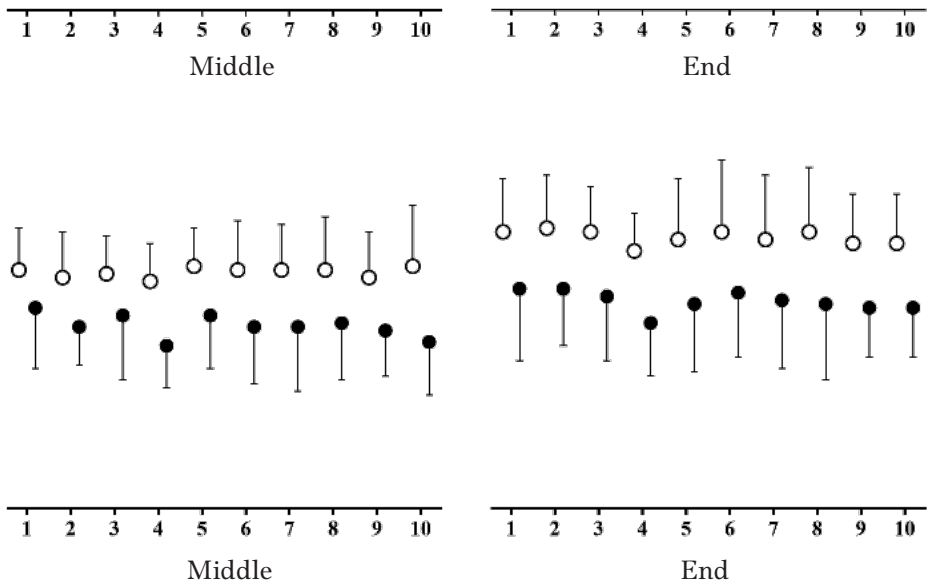

C
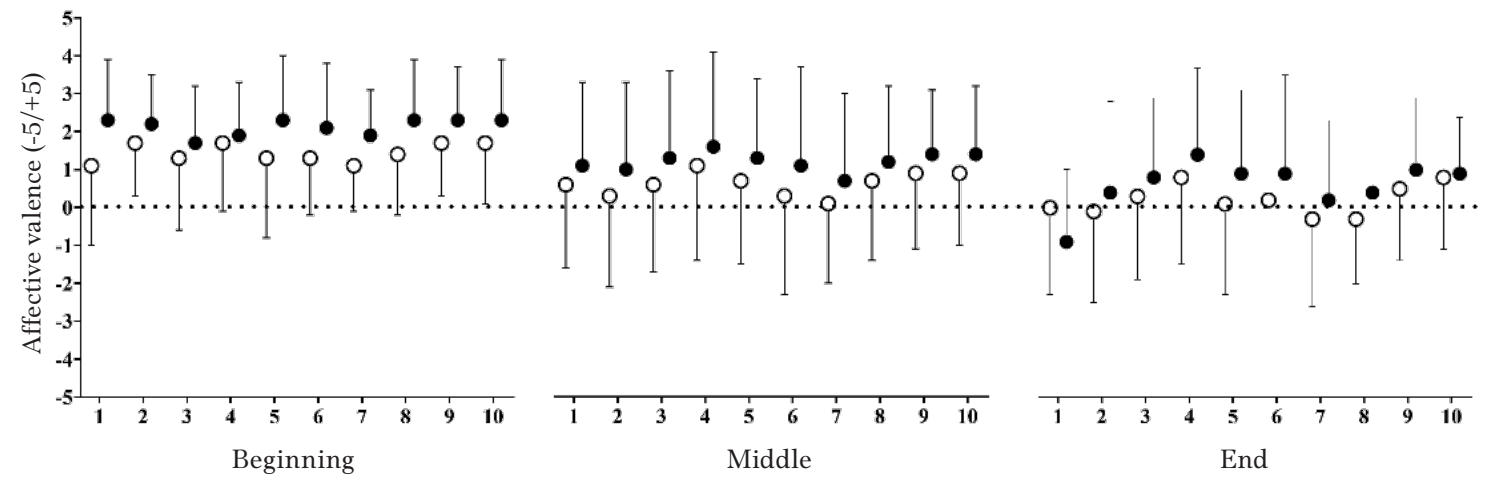

O Intervals

- Recovery

Figure 2. Intensity of exercise (panel A), rating of perceived exertion (panel B), and affective valence (panel C) responses throughout the 10 running high-intensity interval training sessions in the beginning, middle, and end parts.

Data expressed as mean \pm standard deviation 
I.K. Santos et al., Training responses of women with polycystic ovary syndrome

There was a main effect of session parts for HR of intervals [W(2) $=34.509 ; p<0.001]$ and recoveries $[\mathrm{W}(2)=63.306 ; p<0.001]$. For intervals, the HR response at the beginning was $1.8 \%$ (95\% CI: $1.1-2.6 \%$; $p<0.001 ; E S=1.05)$ and $2.9 \%$ (95\% CI: $1.7-4.1 \% ; p$ $<0.001 ; E S=1.64$ ) lower compared with the middle and end, respectively. HR in the middle was $1.1 \%$ (95\% CI: $0.2-2.0 \% ; p<0.001$; ES $=1.49$ ) lower compared with the end. For recovery, the HR at the beginning was 2.8\% (95\% CI: $1.6-4.0 \% ; p<0.001 ; E S$ $=1.18$ ) and 3.6\% (95\% CI: $2.4-4.7 \%$; $p<0.001 ; E S=$ 1.34) lower compared with the middle and end, respectively. HR in the middle was $0.8 \%$ (95\% CI: 0.1$1.4 \% ; p=0.011 ; E S=1.29$ ) lower compared with the end. In all session parts, the participants presented a small coefficient of variation (ca. 3\%) in the HR response of interval and recovery throughout the 10 sessions.

Regarding RPE, there was a main effect of session parts of intervals $[\mathrm{W}(2)=84.407 ; p<0.001]$ and recovery $[\mathrm{W}(2)=41.108 ; p<0.001]$. For intervals, RPE at the beginning was by 1.6 a.u. (95\% CI: $1.0-2.2$ a.u.; $p<0.001 ; E S=0.96)$ and 2.5 a.u. (95\% CI: $1.7-3.3$ a.u.; $p<0.001 ; E S=1.74)$ lower compared with the middle and end, respectively. RPE in the middle was by 0.9 a.u. (95\% CI: 0.7-1.2 a.u.; $p<0.001 ; E S=1.42$ ) lower compared with the end. For recovery, RPE at the beginning was by 1.8 a.u. ( $95 \%$ CI: $1.1-2.5$ a.u.; $p<0.001 ; E S=1.18)$ and 2.4 a.u. (95\% CI: 1.5-3.4 a.u.; $p<0.001 ; E S=1.34)$ lower compared with the middle and end, respectively. RPE in the middle was by 0.6 a.u. (95\% CI: $0.3-1.0$ a.u.; $p=0.011 ; E S=1.29$ ) lower compared with the end. In all session parts, the participants presented a medium coefficient of variation (ca. 20\%) in RPE of interval and recovery throughout the 10 sessions.

As for the affective valence, there was a main effect of session parts of intervals [W(2) $=6.459 ; p=0.040$ ] and recovery $[\mathrm{W}(2)=19.727 ; p<0.001]$. For intervals, affective valence was by 1.2 a.u. higher only at the beginning compared with the end (95\% CI: 0.6-2.3 a.u.; $p=0.035 ; E S=0.63$ ), while there was no difference in affective response between the middle and the end $(p=0.121)$. For recovery, affective response at the beginning was by 1.5 a.u. ( $95 \%$ CI: $0.4-2.6$ a.u.; $p=0.003$; $E S=0.48$ ) higher than at the end, without a difference between the beginning and the middle $(p=0.140)$. Moreover, affective response at the middle was by 0.6 a.u. (95\% CI: $0.2-1.0$ a.u.; $p=0.002$; $E S=0.96$ ) higher than at the end. The coefficient of variation for affective valence was not calculated because of negative values reported by participants in all session parts in interval and recovery.
Figure 2 shows the intensity of exercise, RPE, and affective responses throughout the 10 running HIIT sessions.

\section{Discussion}

This study described the psychophysiological responses (i.e., RPE and affective valence) of physically inactive women with PCOS throughout 10 running HIIT sessions. The main findings in the study were as follows: (i) the physically inactive and overweight women with PCOS presented lower affective responses in the middle and at the end of the sessions compared with the beginning; (ii) the RPE increased and affective response decreased during the HIIT sessions; (iii) the participants' HR and RPE responses presented a small (ca. 3\%) and medium (ca. 20\%) variation, respectively, throughout the 10 running HIIT sessions.

Our results show that psychophysiological responses of physically inactive women with PCOS to running HIIT with 1:3 interval/recovery ratio is session-partdependent, but the affective valence does not become negative. Affective valence was positive from the beginning to the middle of sessions and became neutral at the end of sessions. A reduction of affective valence during HIIT sessions was previously demonstrated by Decker and Ekkekakis [23] with a different HIIT protocol in less active obese women $(4 \times 3$-min intervals of recumbent cycling at $115 \%$ of ventilatory threshold, $4 \times 2$-min periods of active recovery at $85 \%$ of ventilatory threshold). Decker and Ekkekakis [23] used a lower intensity for interval and recovery but a higher interval/ recovery ratio (i.e., 3:2 ratio) in comparison with the interval/recovery ratio of 1:3 that we applied. Therefore, regardless of the interval/recovery ratio and intensity of interval and recovery, physically inactive overweight women present a reduction of affective valence at the end of HIIT sessions, but the training becomes more tolerable with the protocol performed with a lower interval/recovery ratio. An HIIT session with longer intervals (i.e., $>1 \mathrm{~min}$ ) and an interval/recovery ratio $\geq 1$ substantially increases the metabolic imbalance (i.e., anaerobic glycolytic energy, accumulated oxygen deficit, and blood lactate concentration) [1], while a recovery duration greater than 3 min may accelerate blood lactate clearance [1]. Thus, since the affective response is extremely influenced by the metabolic imbalance [39], the prescription of running HIIT for physically inactive overweight women with PCOS should consider the intensity of intervals and recovery, as well as the interval/recovery ratio in order to elicit positive affective valence at the end of the session. 
In our study, the women with PCOS attended all HIIT running sessions (the attendance rate was 100\%) and no adverse events occurred. This is important because the practice of regular exercise is an essential support for treatments in order to achieve improvement in clinical parameters related to PCOS [46]. It is worth noting that observational studies showed a positive correlation between affective valence during moderateintensity exercise and future physical activity [20-22]. In this sense, if physically inactive overweight women with PCOS feel that running HIIT sessions are tolerable, this may influence their physical activity practice in the future. This is supported by the hedonic theory that postulates that repetition of a behaviour is dependent on the pleasure or displeasure that the individual feels during practice [19, 22, 47]. It is of considerable significance as in women with PCOS, high-intensity aerobic exercise promotes improvement of insulin resistance [15], which is a main metabolic disorder that increases the risk of diabetes mellitus and cardiovascular disease in PCOS patients [7]. Therefore, this running HIIT protocol may be prescribed for women with PCOS despite the reduction of affective valence at the end of the session.

Regarding the applicability of the running HIIT protocol used here, first, it was performed on an outdoor 400-m running track. Outdoor exercise promotes higher external attention focus, positive effects, and lower RPE, followed by greater enjoyment and stronger future intention in women with obesity [48]. Second, the participants were instructed in regard to time and intensities of intervals and recovery, as well as how to carry out the exercises (i.e., running and walking), and did so accordingly. Together, these simple instructions make the running HIIT protocol applicable. The participants' HR response to intervals and recovery was achieved, as expected, with a small coefficient of variation (ca. 3\%). Additionally, the RPE matched with the high intensity initially proposed with a medium coefficient of variation (ca. 20\%). However, it is important to highlight that we used a supervised approach, which had been previously demonstrated as more efficient than an unsupervised approach for less active individuals [49]. Therefore, it is crucial that future studies investigate the effectiveness of an unsupervised protocol in women with PCOS to promote improvement in health parameters and an increase in physical activity level.

Another important component to mention is the frequency and adherence to an aerobic exercise program. These intervention programs can facilitate adherence, defined as the number of sessions attended, and compliance with the prescribed intensity and duration of the training sessions [50]; participation, in turn, can contribute to a program's high effectiveness [51]. In this context, adherence to the interventions of exercises prescribed during treatment, in addition to being a challenge, is fundamental to understand the changes and the results [52].

Despite these interesting findings, some limitations need to be mentioned. First, we used the formula for calculating HRmax to prescribe the intensities of the HIIT session instead of a parameter identified by a maximal incremental test or the use of HR reserve as recommended. Second, the sample of this study included ca. $40 \%$ of normal weight women, so it is possible that the results were influenced by the different nutritional status of the participants. Third, there was no comparison group.

From a psychophysiological perspective, this study suggests the running HIIT protocol used here as a potential approach of high-intensity aerobic exercise for treatment of women with PCOS, since some methodological precautions were followed favouring the ecological validity of the study.

\section{Conclusions}

In summary, physically inactive women with PCOS present positive affective valence at the beginning of HIIT sessions; however, throughout the session, the affective valence decreases to neutral. The HIIT protocol with an interval/recovery ratio of $1: 3$ is more tolerable for physically inactive women with PCOS, especially at the end of the session. Given the importance of PCOS patients' participation in interventions of lifestyle modifications such as physical exercises, future investigations are necessary into the effectiveness of this protocol to promote adherence to exercise, as well as improvement in cardiometabolic risk factors and quality of life in women with PCOS.

\section{Acknowledgements}

The authors would like to thank all the study participants.

\section{Disclosure statement}

No author has any financial interest or received any financial benefit from this research.

\section{Conflict of interest}

The authors state no conflict of interest. 
I.K. Santos et al., Training responses of women with polycystic ovary syndrome

\section{Funding}

The Coordenação de Aperfeiçoamento de Pessoal de Nível Superior (CAPES), Brazil, provided support for the PhD scholarship of the first author (Finance Code 001) and the postdoctoral fellowships of the second author.

\section{References}

1. Buchheit M, Laursen PB. High-intensity interval training, solutions to the programming puzzle. Part II: anaerobic energy, neuromuscular load and practical applications. Sports Med. 2013;43(10):927-954; doi: 10.1007/s40279-013-0066-5.

2. Gibala MJ, Little JP, MacDonald MJ, Hawley JA. Physiological adaptations to low-volume, high-intensity interval training in health and disease. J Physiol. 2012;590(5):1077-1084; doi: 10.1113/jphysiol.2011. 224725.

3. Keating SE, Johnson NA, Mielke GI, Coombes JS. A systematic review and meta-analysis of interval training versus moderate-intensity continuous training on body adiposity. Obes Rev. 2017;18(8):943-964; doi: 10.1111/obr.12536.

4. Batacan RB, Duncan MJ, Dalbo VJ, Tucker PS, Fenning AS. Effects of high-intensity interval training on cardiometabolic health: a systematic review and meta-analysis of intervention studies. Br J Sports Med. 2017;51(6):494-503; doi: 10.1136/bjsports-2015095841.

5. Caldas Costa E, Ferezini de Sá JC, Stepto NK, Bezerra Barbosa Costa I, Farias-Junior LF, Da Nóbrega Tomaz Moreira S, et al. Aerobic training improves quality of life in women with polycystic ovary syndrome. Med Sci Sports Exerc. 2018;50(7):1357-1366; doi: 10.1249/ MSS.0000000000001579.

6. Dos Santos IK, de Souza Nunes FAS, Queiros VS, Cobucci RN, Bruch Dantas P, Mafaldo Soares G, et al. Effect of high-intensity interval training on metabolic parameters in women with polycystic ovary syndrome: a systematic review and meta-analysis of randomized controlled trials. PLoS One. 2021;16(1):e0245023; doi: 10.1371/journal.pone.0245023.

7. Almenning I, Rieber-Mohn A, Lundgren KM, Shetelig Løvvik T, Krohn Garnæs K, Moholdt T. Effects of high intensity interval training and strength training on metabolic, cardiovascular and hormonal outcomes in women with polycystic ovary syndrome: a pilot study. PLoS One. 2015;10(9):e0138793; doi: 10.1371/journal.pone.0138793.

8. Bozdag G, Mumusoglu S, Zengin D, Karabulut E, Yildiz BO. The prevalence and phenotypic features of polycystic ovary syndrome: a systematic review and metaanalysis. Hum Reprod. 2016;31(12):2841-2855; doi: 10.1093/humrep/dew218.

9. Kakoly NS, Khomami MB, Joham AE, Cooray SD, Misso ML, Norman RJ, et al. Ethnicity, obesity and the prevalence of impaired glucose tolerance and type 2 diabetes in PCOS: a systematic review and meta-regression. Hum Reprod Update. 2018;24(4):455-467; doi: 10.1093/humupd/dmy007.

10. Le MT, Nguyen VQH, Truong QV, Le DD, Le VNS, Cao NT. Metabolic syndrome and insulin resistance syndrome among infertile women with polycystic ovary syndrome: a cross-sectional study from Central Vietnam. Endocrinol Metab. 2018;33(4):447-458; doi: 10.3803/EnM.2018.33.4.447.

11. Teede HJ, Misso ML, Costello MF, Dokras A, Laven J, Moran L, et al. Recommendations from the international evidence-based guideline for the assessment and management of polycystic ovary syndrome. Fertil Steril. 2018;110(3):364-379; doi: 10.1016/j.fertnstert.2018.05. 004.

12. Dos Santos IK, Ashe MC, Cobucci RN, Mafaldo Soares G, de Oliveira Maranhão TM, Moreira Silva Dantas P. The effect of exercise as an intervention for women with polycystic ovary syndrome: a systematic review and meta-analysis. Medicine. 2020;99(16): e19644; doi: 10.1097/MD.0000000000019644.

13. Benham JL, Yamamoto JM, Friedenreich CM, Rabi DM, Sigal RJ. Role of exercise training in polycystic ovary syndrome: a systematic review and meta-analysis. Clin Obes. 2018;8(4):275-284; doi: 10.1111/cob.12258.

14. Moran LJ, Hutchison SK, Norman RJ, Teede HJ. Lifestyle changes in women with polycystic ovary syndrome. Cochrane Database Syst Rev. 2011;2:CD007506; doi: 10.1002/14651858.CD007506.pub2.

15. Harrison CL, Stepto NK, Hutchison SK, Teede HJ. The impact of intensified exercise training on insulin resistance and fitness in overweight and obese women with and without polycystic ovary syndrome. Clin Endocrinol. 2012;76(3):351-357; doi: 10.1111/j.1365-2265. 2011.04160.x.

16. Samadi Z, Bambaeichi E, Valiani M, Shahshahan Z. Evaluation of changes in levels of hyperandrogenism, hirsutism and menstrual regulation after a period of aquatic high intensity interval training in women with polycystic ovary syndrome. Int J Prev Med. 2019;10(1): 187; doi: 10.4103/ijpvm.IJPVM_360_18.

17. Aktaş HŞ, Uzun YE, Kutlu O, Pençe HH, Özçelik F, Çil EÖ, et al. The effects of high intensity-interval training on vaspin, adiponectin and leptin levels in women with polycystic ovary syndrome. Arch Physiol Biochem. 2019;1-6; doi: 10.1080/13813455.2019.1662450.

18. Patten RK, Boyle RA, Moholdt T, Kiel I, Hopkins WG, Harrison CL, et al. Exercise interventions in polycystic ovary syndrome: a systematic review and meta-analysis. Front Physiol. 2020;11:606; doi: 10.3389/fphys. 2020.00606.

19. Garber CE, Blissmer B, Deschenes MR, Franklin BA, Lamonte MJ, Lee I-M, et al. American College of Sports Medicine position stand. Quantity and quality of exercise for developing and maintaining cardiorespiratory, musculoskeletal, and neuromotor fitness in apparently 
healthy adults: guidance for prescribing exercise. Med Sci Sports Exerc. 2011;43(7):1334-1359; doi: 10.1249/ MSS.0b013e318213fefb.

20. Williams DM, Dunsiger S, Jennings EG, Marcus BH. Does affective valence during and immediately following a 10-min walk predict concurrent and future physical activity? Ann Behav Med. 2012;44(1):43-51; doi: 10.1007/s12160-012-9362-9.

21. Williams DM, Dunsiger S, Ciccolo JT, Lewis BA, Albrecht $\mathrm{AE}$, Marcus $\mathrm{BH}$. Acute affective response to a moderate-intensity exercise stimulus predicts physical activity participation 6 and 12 months later. Psychol Sport Exerc. 2008;9(3):231-245; doi: 10.1016/j.psychsport.2007.04.002.

22. Rhodes RE, Kates A. Can the affective response to exercise predict future motives and physical activity behavior? A systematic review of published evidence. Ann Behav Med. 2015;49(5):715-731; doi: 10.1007/ s12160-015-9704-5.

23. Decker ES, Ekkekakis P. More efficient, perhaps, but at what price? Pleasure and enjoyment responses to high-intensity interval exercise in low-active women with obesity. Psychol Sport Exerc. 2017;28:1-10; doi: 10.1016/j.psychsport.2016.09.005.

24. Ekkekakis P, Lind E, Vazou S. Affective responses to increasing levels of exercise intensity in normal-weight, overweight, and obese middle-aged women. Obesity. 2010;18(1):79-85; doi: 10.1038/oby.2009.204.

25. Hardcastle SJ, Ray H, Beale L, Hagger MS. Why sprint interval training is inappropriate for a largely sedentary population. Front Psychol. 2014;5:1505; doi: 10.3389/fpsyg.2014.01505.

26. Ekkekakis P. The dual-mode theory of affective responses to exercise in metatheoretical context: I. Initial impetus, basic postulates, and philosophical framework. Int Rev Sport Exerc Psychol. 2009;2(1):73-94; doi: 10.1080/17509840802705920.

27. Farias-Junior LF, Browne RAV, Astorino TA, Costa EC. Physical activity level and perceived exertion predict intask affective valence to low-volume high-intensity interval exercise in adult males. Physiol Behav. 2020;224: 112960; doi: 10.1016/j.physbeh.2020.112960.

28. Farias-Junior LF, Browne RAV, Freire YA, OliveiraDantas FF, Araújo Moura Lemos TM, Leite Galvão-Coelho N, et al. Psychological responses, muscle damage, inflammation, and delayed onset muscle soreness to high-intensity interval and moderate-intensity continuous exercise in overweight men. Physiol Behav. 2019; 199:200-209; doi: 10.1016/j.physbeh.2018.11.028.

29. Frazão DT, de Farias Junior LF, Dantas TCB, Krinski K, Elsangedy HM, Prestes J, et al. Feeling of pleasure to high-intensity interval exercise is dependent of the number of work bouts and physical activity status. PLoS One. 2016;11(3):e0152752; doi: 10.1371/journal.pone. 0152752 .

30. Knottnerus A, Tugwell P. STROBE - a checklist to strengthen the reporting of observational studies in epidemiology. J Clin Epidemiol. 2008;61(4):323; doi: 10.1016/j.jclinepi.2007.11.006.

31. The Rotterdam ESHRE/ASRM-sponsored PCOS consensus workshop group. Revised 2003 consensus on diagnostic criteria and long-term health risks related to polycystic ovary syndrome. Fertil Steril. 2004;81(1): 19-25; doi: 10.1016/j.fertnstert.2003.10.004.

32. Thomas S, Reading J, Shephard RJ. Revision of the Physical Activity Readiness Questionnaire (PAR-Q). Can J Sport Sci. 1992;17(4):338-345.

33. Craig CL, Marshall AL, Sjöström M, Bauman AE, Booth ML, Ainsworth BE, et al. International Physical Activity Questionnaire: 12-country reliability and validity. Med Sci Sports Exerc. 2003;35(8):1381-1395; doi: 10.1249/01.MSS.0000078924.61453.FB.

34. World Health Organization. Global strategy on diet, physical activity and health: obesity and overweight. Geneva: World Health Organization; 2003.

35. Huxley R, Mendis S, Zheleznyakov E, Reddy S, Chan J. Body mass index, waist circumference and waist:hip ratio as predictors of cardiovascular risk - a review of the literature. Eur J Clin Nutr. 2010;64(1):16-22; doi: 10.1038/ejcn.2009.68.

36. Schuindt da Silva V, Soares Vieira MF. International Society for the Advancement of Kinanthropometry (ISAK) Global: international accreditation scheme of the competent anthropometrist. Rev Bras Cineantropom Desempenho Hum. 2020;22:e70517; doi: 10.1590/ 1980-0037.2020v22e70517.

37. Malachias MVB, Gomes MAM, Nobre F, Alessi A, Feitosa AD, Coelho EB. 7th Brazilian guideline of arterial hypertension: Chapter 2 - Diagnosis and classification. Arq Bras Cardiol. 2016;107(3 Suppl. 3):7-13; doi: 10.5935/abc.20160152.

38. Hardy CJ, Rejeski WJ. Not what, but how one feels: the measurement of affect during exercise. J Sport Exerc Psychol. 1989;11(3):304-317; doi: 10.1123/jsep.11.3.304.

39. Ekkekakis P, Parfitt G, Petruzzello SJ. The pleasure and displeasure people feel when they exercise at different intensities: decennial update and progress towards a tripartite rationale for exercise intensity prescription. Sports Med. 2011;41(8):641-671; doi: 10.2165/11590 680-000000000-00000.

40. Alves ED, Leme Gonçalves Panissa V, Barros BJ, Franchini E, Takito MY. Translation, adaptation, and reproducibility of the Physical Activity Enjoyment Scale (PACES) and Feeling Scale to Brazilian Portuguese. Sport Sci Health. 2019;15(2):329-336; doi: 10.1007/ s11332-018-0516-4.

41. Borg GAV. Psychophysical bases of perceived exertion. Med Sci Sports Exerc. 1982;14(5):377-381; doi: 10.1249/ 00005768-198205000-00012.

42. Lee O, Jung J-W. Validity of Borg's category ratio 10 scale during maximal-graded exercise testing [in Korean]. Exerc Sci. 2016;25(2):92-99; doi: 10.15857/ KSEP.2016.25.2.92. 


\section{HUMAN MOVEMENT}

I.K. Santos et al., Training responses of women with polycystic ovary syndrome

43. Robertson RJ, Noble BJ. Perception of physical exertion: methods, mediators, and applications. Exerc Sport Sci Rev. 1997;25:407-452; doi: 10.1249/00003677-19970 0250-00017.

44. Lakens D. Calculating and reporting effect sizes to facilitate cumulative science: a practical primer for t-tests and ANOVAs. Front Psychol. 2013;4:863; doi: 10.3389/fpsyg.2013.00863.

45. Cohen J. Statistical power analysis for the behavioral sciences. New York: Routledge; 2013.

46. Teede HJ, Misso ML, Deeks AA, Moran LJ, Stuckey BGA, Wong JLA, et al. Assessment and management of polycystic ovary syndrome: summary of an evidencebased guideline. Med J Aust. 2011;195(6):65-112; doi: 10.5694/mja11.10915.

47. Cabanac M. Physiological role of pleasure. Science. 1971; 173(4002):1103-1107; doi: 10.1126/science.173.4002. 1103.

48. Krinski K, Machado DGS, Lirani LS, DaSilva SG, Costa EC, Hardcastle SJ, et al. Let's walk outdoors! Self-paced walking outdoors improves future intention to exercise in women with obesity. J Sport Exerc Psychol. 2017;39(2):145-157; doi: 10.1123/jsep.20160220.

49. Fennell C, Peroutky K, Glickman EL. Effects of supervised training compared to unsupervised training on physical activity, muscular endurance, and cardiovascular parameters. MOJ Orthop Rheumatol. 2016;5(4): 00184; doi: 10.15406/mojor.2016.05.00184.

50. World Health Organization. Adherence to long-term therapies: evidence for action. Geneva: World Health Organization; 2003.

51. Cyarto EV, Brown WJ, Marshall AL. Retention, adherence and compliance: important considerations for home- and group-based resistance training programs for older adults. J Sci Med Sport. 2006;9(5):402-412; doi: 10.1016/j.jsams.2006.06.020.

52. Argent R, Daly A, Caulfield B. Patient involvement with home-based exercise programs: can connected health interventions influence adherence? JMIR Mhealth Uhealth. 2018;6(3):e47; doi: 10.2196/mhealth.8518. 